\title{
A Calculation of the Dynamic Aperture of the LHC at Collision * $\dagger$
}

\author{
Norman M. Gelfand, Fermilab, Batavia, IL
}

\begin{abstract}
The dynamic aperture for version 5.1, with a $300 \mu \mathrm{r}$ crossing angle at IP1 and IP5, of the Large Hadron Collider (LHC) lattice has been estimated using the tracking code TEVLAT. The dynamic aperture calculated here is due to the magnetic field errors in the high gradient quadrupoles (MQX) in the two low- $\beta$ interaction regions at IR1 and IR5. No errors were assigned to the magnets in the arc regions of the lattice, nor were beam-beam effects incorporated. The dynamic aperture is expressed in terms of the $\sigma$ of the beam corresponding to the beam emittance of $3.75 \mathrm{~mm} \mathrm{mr}$. With only short term tracking, the combined effect of the multipoles and the crossing angle is to yield an average (over multipole coefficients generated with 100 different seeds) dynamic aperture of $\approx 11.7 \pm 1.2 \sigma$.
\end{abstract}

\section{INTRODUCTION}

It is necessary with any new lattice, to try and calculate the emittance of the beam that can be accepted by the lattice and to verify that the acceptance, the dynamic aperture, is large enough for the intended beam. The physical aperture of the magnets, of course, sets the absolute upper limit to the dynamic aperture, but with superconducting magnets the dynamic aperture is typically limited by the high order multipoles (the magnets errors) used to describe the field of the magnet.

The methodology for calculating the dynamic aperture is fairly standard:

- Construct a computer model of the lattice;

- Adjust the tunes and chromaticites with the arc quadrupoles and sextupoles to the nominal values;

- Introduce the magnetic field errors;

- Track a series of test particles for a given number of turns;

- Impose an aperture limit of $\pm 30 \mathrm{~mm}$; and

- Calculate the emittance of the test particles which survive for the required number of turns.

While well defined, this procedure can only give an upper limit to the dynamic aperture since,

- The model of the lattice is an approximation to the true lattice and all the unknown errors which exist in an actual lattice are not incorporated in to the model.

\footnotetext{
* Work supported by the U.S. Department of Energy under contract No. DE-AC02-76CHO3000.

† Email: gelfand@popgtw.fnal.gov
}

- The actual magnet errors are not known until the magnets are built and measured.

- A particle can be realistically tracked for a number of turns which is far smaller than the number of turns it will need to survive in the actual lattice.

With these caveats, results are presented on the dynamic aperture for version 5.1 the Large Hadron Collider (LHC) lattice. The lattice has the beams crossing at IR1 and IR5 with a $300 \mu \mathrm{r}$ full crossing angle. At IR1 the crossing angle is vertical and at IR5 horizontal. Tracking was done using the code TEVLAT [1].

The lattice has regions of very high- $\beta(\approx 4700 \mathrm{~m})$ in the inner triplet quadrupoles (MQX), in which the beam is off axis, which generate the low- $\beta$ at the interaction points. The corresponding maximum $\beta$ in the arcs is only $\approx 180 \mathrm{~m}$. The effect of magnetic field errors increase with $\beta$ so it is expected that the errors in the MQX will dominate the calculation of the dynamic aperture. For that reason only the magnetic field errors in the MQX will be considered and incorporated into this calculation.

\section{MAGNET ERRORS}

The low- $\beta$ insertions at each IR are formed by a quadrupole triplet consisting of four quadrupoles [2]. Two different designs of the coils for the MQX will be used in the LHC, those built at FERMILAB and those built at KEK. In the model the outer members of the triplet are KEK magnets while the two inner magnets are from FERMILAB. The field errors for each are expressed in terms of multipole coefficients given at a reference radius of $17 \mathrm{~mm}$. The values for the multipole coefficients are given in Table 1 and Table 2.[3]. One hundred different sets of multpole errors were generated from these tables.

Table 1

FNAL Reference Harmonics at Collision. (Version 2) Reference radius $=17.0 \mathrm{~mm}$

\begin{tabular}{|c|r|r|r|r|}
\hline $\begin{array}{c}\text { CERN } \\
\mathrm{n}\end{array}$ & $\left\langle b_{n}\right\rangle$ & $\sigma\left(b_{n}\right)$ & $\left\langle a_{n}\right\rangle$ & $\sigma\left(a_{n}\right)$ \\
\hline 3 & .000 & .800 & .000 & .800 \\
4 & .000 & .800 & .000 & .800 \\
5 & .000 & .300 & .000 & .300 \\
6 & .000 & .600 & .000 & .100 \\
7 & .000 & .060 & .000 & .060 \\
8 & .000 & .050 & .000 & .040 \\
9 & .000 & .030 & .000 & .020 \\
10 & .000 & .030 & .000 & .030 \\
\hline
\end{tabular}


Table 2

KEK Reference Harmonics at Collision. (Modified.) Reference radius $=17.0 \mathrm{~mm}$

\begin{tabular}{|c|r|r|r|r|}
\hline $\begin{array}{c}\text { CERN } \\
\mathrm{n}\end{array}$ & $\left\langle b_{n}\right\rangle$ & $\sigma\left(b_{n}\right)$ & $\left\langle a_{n}\right\rangle$ & $\sigma\left(a_{n}\right)$ \\
\hline 3 & .000 & 1.00 & .000 & 1.00 \\
4 & .000 & .570 & .000 & .570 \\
5 & .000 & .380 & .000 & .380 \\
6 & .000 & .190 & .000 & .190 \\
7 & .000 & .060 & .000 & .060 \\
8 & .000 & .030 & .000 & .010 \\
9 & .000 & .010 & .000 & .010 \\
10 & -0.250 & .010 & .000 & .010 \\
\hline
\end{tabular}

\section{METHOD AND RESULTS}

A particle was launched at a point in phase space $\left(x, x^{\prime}=\right.$ $0),\left(y, y^{\prime}=0\right)$. For each value of $x$, which ranged from $x=$ 0 to $x=20 \cdot \sigma_{x}$ [4] in steps of $\sigma_{x}$, the maximum value of $\mathrm{y}$ was found where the particle completed 1024 turns. The stable points, from the hundred sets of multipole errors, are plotted in Figure 1.

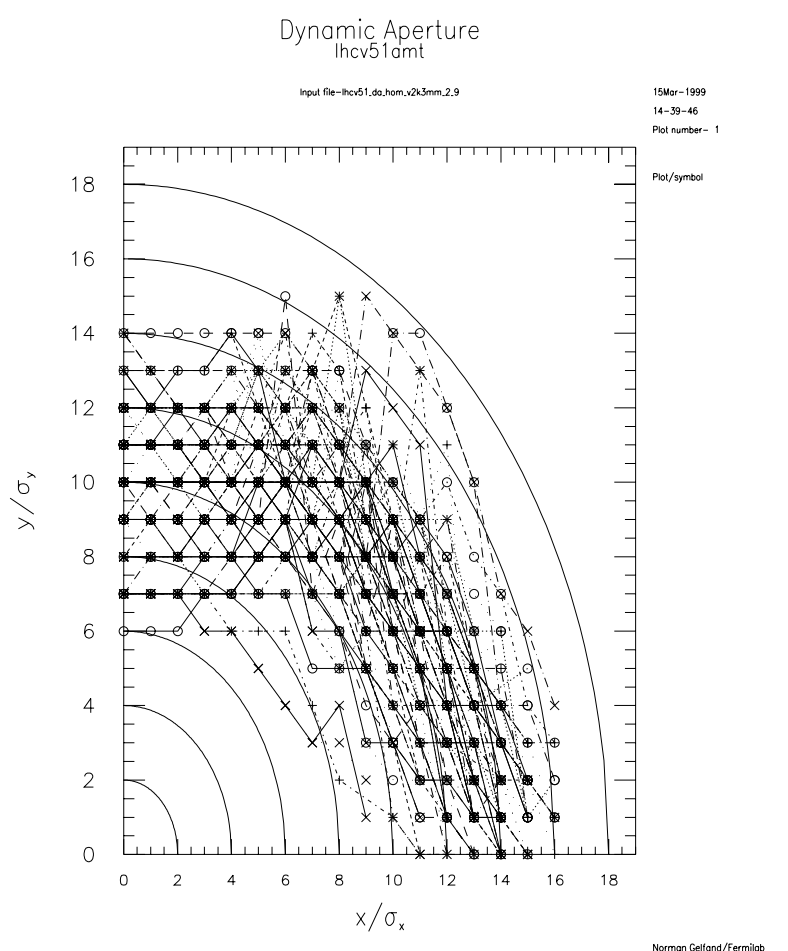

Figure 1: Stable Points for 100 Different Sets of Multipole Errors.

It is apparent that the dynamic aperture depends on the particular values of the multipole coefficients. That is, the size of the random component of the multipoles is large enough that the dynamic aperture can change by a significant factor depending on the value in a given magnet.
In order to provide a more concise representation of the dynamic aperture, (though a less complete one) for each set of the multipoles errors, the average radius of the stable points was computed. The results are histogrammed in Figure 2 [5].

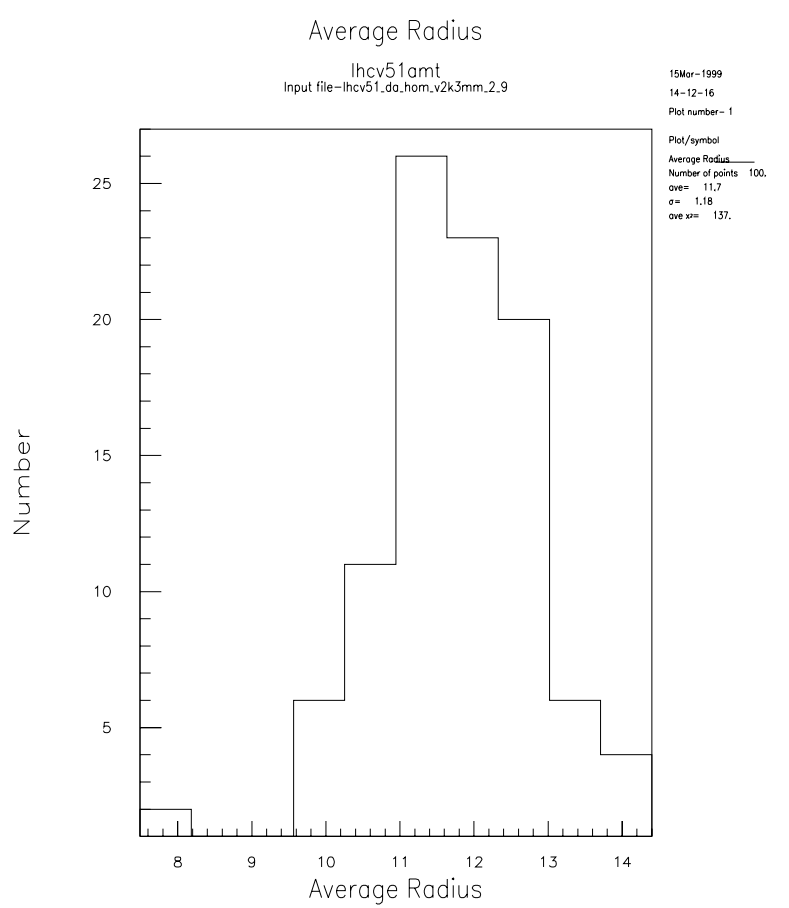

Figure 2: Distribution of the Radius for the Stable Points

The average dynamic aperture, that is the average of the average radius for the 100 distributions is $11.7 \sigma$ with a width of $\approx 1.2 \sigma$. Depending on the set of multipoles found in the actual magnets the dynamic aperture could be as small as $\approx 8 \sigma$ or as large as $\approx 14 \sigma$.

The dynamic aperture also depends on whether the initial displacement was primarily along $x$ or along $y$. Figure 3 plots the average dynamic aperture as a function of $\epsilon_{x} /\left(\epsilon_{x}+\epsilon_{y}\right)$. It is quite clear that the dynamic aperture, when a particle is launched along the $y$ direction, is smaller than if it were launched along the $x$ direction.

It is helpful to compute the dynamic aperture with only some of the multipoles present. Figure 4 shows the effect on the dynamic aperture when the highest order of the multipoles is changed. The results are summarized in Table 3.

If it were possible to remove the $b_{10}$ and $a_{10}$ multipole errors from the magnets we would gain $\approx 1.6 \sigma$ in the dynamic aperture. There are no other significant improvements until we remove the $b_{6}$ and $a_{6}$ from the magnets.

The variance of the distribution of the dynamic apertures decreases when the octupole contribution to the magnetic field is removed. The octupole moment contributes to an amplitude dependent tune shift. A smaller random octupole moment would reduce the spread in the dynamic aperture 


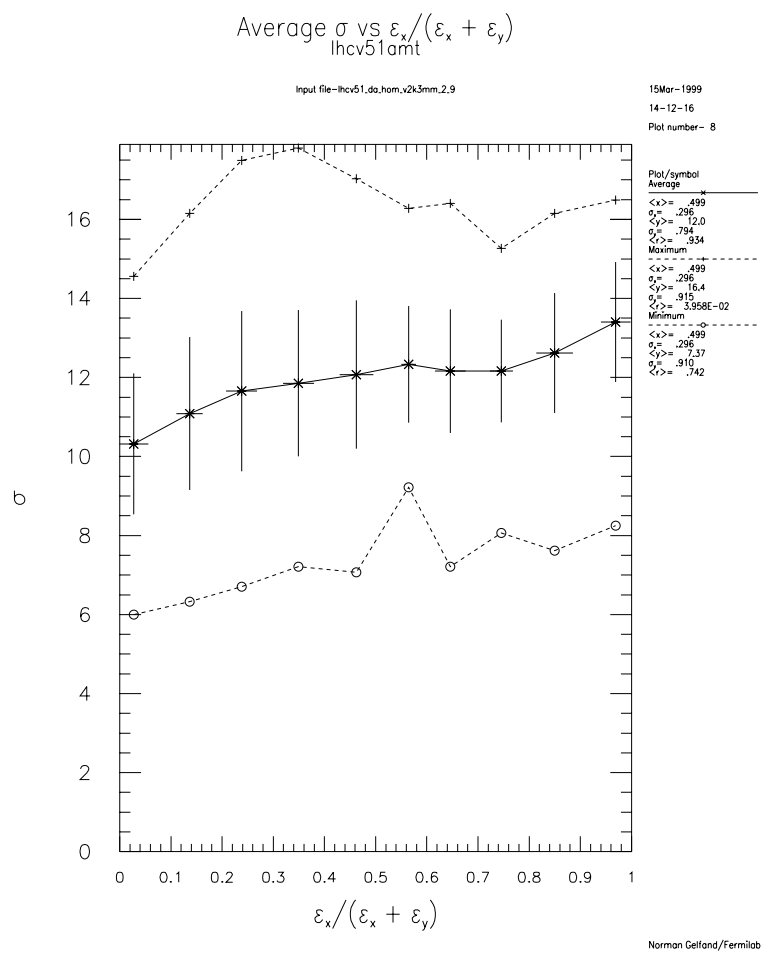

Figure 3: Dynamic Aperture vs. $\epsilon_{x} /\left(\epsilon_{x}+\epsilon_{y}\right)$ The upper and lower curves are the maximum and minimum values coming from the different sets of the moments.

Table 3

The Effect of Removing High Order Multipoles on the Dynamic Aperture

\begin{tabular}{|c|c|r|r|}
\hline Min. Order & Max. Order & Average DA & Variance \\
\hline 3 & 10 & 11.659 & 1.181 \\
3 & 9 & 13.396 & 1.551 \\
3 & 8 & 13.446 & 1.533 \\
3 & 7 & 13.459 & 1.518 \\
3 & 6 & 13.530 & 1.554 \\
3 & 5 & 14.147 & 1.272 \\
3 & 4 & 14.576 & 1.032 \\
3 & 3 & 15.199 & .867 \\
\hline
\end{tabular}

seen in Figure 1 and Figure 2.

\section{CONCLUSION}

This is the first use of TEVLAT for calculations on the LHC lattice, though it has been used extensively with other lattices. The calculations presented here utilize 100 different sets of multipole error coefficients, a factor of 10 more than calculations reported earlier. The large variation in the dynamic aperture, including tails in the distribution, makes it important to do calculations with a large number of sets of multipole coefficients for the MQX. This variance suggests a need to monitor the random component of the magnet er-

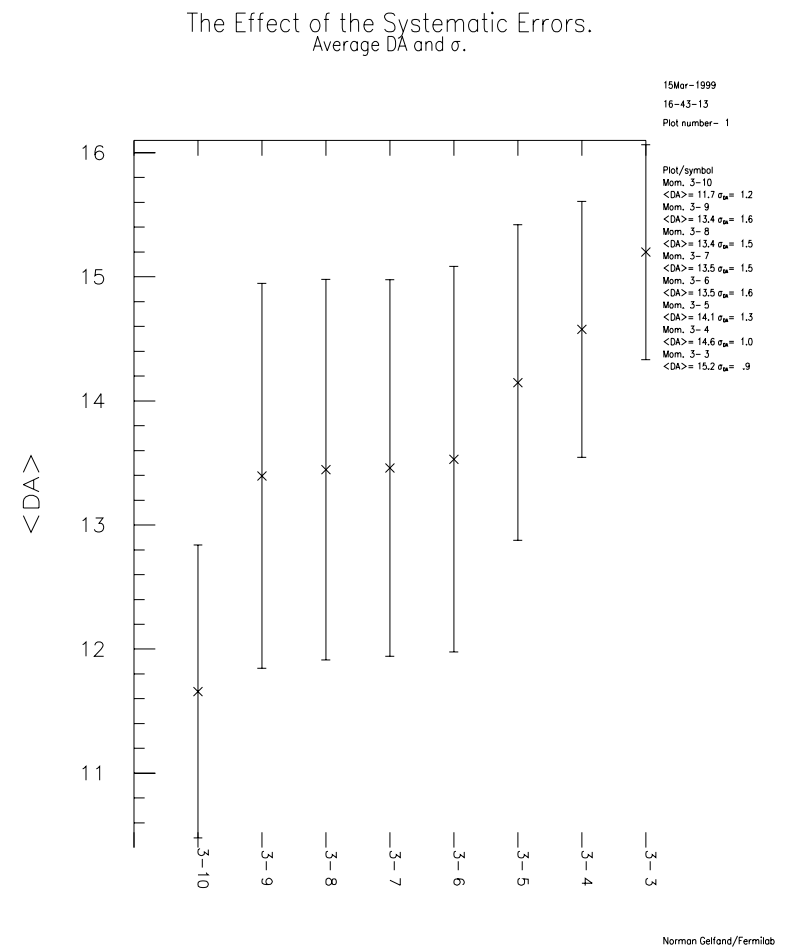

Figure 4: The Variation in the Dynamic Aperture with the Order of the Multipoles

rors, particularly the octupoles, since they make a significant contribution to the computed variance in the dynamic aperture. Non-linear correctors could also be used to reduce the variance.

The dynamic aperture appears sensitive to the highest order multipole specified in the reference tables. Tracking has also been done with errors in all magnets derived from an earlier version of the Fermilab error table. This error table included estimates for the moments up to $b_{14}$ and $a_{14}$. Those calculations showed a decrease in the dynamic aperture when the moments beyond $b_{10}$ were included. It is important to check that the dynamic aperture is not sensitive to the multipoles which will be present in the magnets, but because they are not specified, are not included in the calculations described here.

\section{REFERENCES}

[1] A. Russell, private communication.

[2] The two middle magnets are used as a single optical element.

[3] The uncertainty in the average multipoles, $d b_{n}$ and $d a_{n}$ were not included in the calculations.

[4] $\sigma_{x}$ and $\sigma_{y}$ are defined by the values of the lattice functions at the launch point and the normalized invariant emittance of the beam. For the LHC $\epsilon_{n x}=\epsilon_{n y}=3.75 \mathrm{mmmr}$.

[5] The radius of a stable point is defined as $r=\sqrt{\left.\left(x / \sigma_{x}\right)^{2}+\left(y / \sigma_{y}\right)^{2}\right)}$ 